\title{
HEALTH VERSUS APPEARANCE FOCUS IN ONE- VERSUS TWO-SIDED MESSAGES DISCOURAGING SUN TANNING
}

Erlinde Cornelis, Ghent University, Belgium

Veroline Cauberghe, Ghent University, Belgium

Patrick De Pelsmacker, University of Antwerp, Belgium

Corresponding author:

Erlinde Cornelis

Ghent University

Korte Meer 7-9-11

9000 Gent

Belgium

Email: erlinde.cornelis@ugent.be

Tel: +32092646868

Fax: 003292646992

The first author (a PhD student) is nominated for the Best Student Paper Award 


\title{
HEALTH VERSUS APPEARANCE FOCUS IN ONE- VERSUS TWO-SIDED MESSAGES DISCOURAGING SUN TANNING
}

\begin{abstract}
In a 2 by 2 experimental design, this study investigates the impact of message sidedness (oneversus two-sided) and argument focus (health versus appearance) in messages to discourage sun tanning. The results of this study show that two-sided anti sun tanning messages receive more attention and generate a higher message content recall than their conventional one-sided counterparts. Additionally, when a message is two-sided, appearance focused arguments lead to lower behavioral intentions to suntan than health focused arguments, whereas for a onesided messages, health versus appearance focus has no different impact.
\end{abstract}

\section{INTRODUCTION}

Despite widespread awareness of the negative consequences of unprotected UV exposure, sun tanning $^{1}$ is still a common practice among many people in western countries (Heckman, Wilson, \& Ingersoll, 2009). Sun tanning increases the risks of skin cancer and photo-aging (Hoegh et al., 1999; Turrisi et al., 1998). Consistent findings across studies show that most people have sufficient knowledge of these risks, but this knowledge does not always transfer into behavioral intentions to limit UV exposure (Arthey \& Clarke, 1995). Hence, there is still a strong need for adequate prevention campaigns.

To date, most health prevention campaigns aim to increase risk awareness of several unhealthy behaviors (e.g., binge drinking, smoking, or sun tanning) among people, by emphasizing the risks associated with certain unhealthy behavior. However, this mere focus on the negative aspects of unhealthy behavior is rather one-sided. Overuse of such one-sided negative appeal can lead to habituation, possibly evoking a saturation effect (Ahn et al., 2011; Devlin et al., 2007). The present study assesses an alternative and potentially more persuasive way of communicating health risks: two-sided messages, "in which the communicator takes into account both sides of an issue, but actually still favors one side" (Hovland, 1954). A variety of studies show that commercial advertising messages can include some negative information along with the positive product information, and can still be more effective than when only positive information is provided (Crowley \& Hoyer, 1994; Pechmann, 1992). However, the two-sided message strategy is mainly applied to commercial product advertising (Eisend, 2006). A few notable studies applied two-sided messages to health communication (e.g., Belch, 1981 selling toothpaste; Ford \& Smith, 1991 promoting organ donation; Ley et al., 1977 promoting weight loss), but - to our knowledge - this principle has not been tested in a reversed way (i.e., to discourage unhealthy behavior). The current study investigates twosided messages in a reversed way by focusing on the negative consequence of certain unhealthy behavior while also mentioning a minor positive aspect of that behavior, which often is the main motivator to perform the unhealthy behavior.

Often, individuals engage in unhealthy behaviors such as smoking, eating junk food, or sun tanning, because they perceive certain benefits or pleasures of this behavior. These perceived benefits often impede behavior change. Western studies have shown that sun tanning is primarily done because people believe a tanned skin enhances their attractiveness (Heckman et al., 2009). Literature suggests that appearance-related motivations are the primary reason for sun tanning (Heckman et al., 2009; Jones \& Leary, 1994; Turrisi et al., 1998). However,

\footnotetext{
${ }^{1}$ In the present study, sun tanning refers to the intentional exposure to UV light in general, that is both natural (sunlight) as well as artificial (tanning beds) UV light.
} 
most of the previous skin cancer prevention literature focuses on health-related, instead of appearance-related attributes to provoke attitude changes. More recent studies show that such tanning interventions, solely focusing on health aspects (such as skin cancer) without a focus on appearance, may fall short (Heckman et al., 2009).

Hence, this study uses a $2 \times 2$ between-subjects factorial experimental design, in which we investigate the effectiveness of health versus appearance focused one- and two-sided messages on individuals' intentions to suntan.

\section{THEORETICAL FRAMEWORK AND HYPOTHESIS DEVELOPMENT}

Ample previous studies comparing one- versus two-sided messages were based on dual processing theories, such as the elaboration likelihood model (Eisend, 2007). According to these theories, two-sided messages - incorporating both sides of an issue - would be perceived as more unusual (or less conventional) than one-sided messages, thereby motivating individuals to pay more attention to the message (Eisend, 2006, 2007). In contrast, one-sided messages represent the type of communication that the individual is normally expecting, resulting in relatively less attention towards the message (Crowley \& Hoyer, 1994). Eisend (2006) confirmed these expectations in his meta-analysis. Hence, we expect that:

\section{H.1 A two-sided anti sun tanning message attracts more attention than a one-sided anti sun tanning message.}

Previous research established that two-sided messages are more likely to trigger elaborate, indepth processing than one-sided messages (Crowley \& Hoyer, 1994; Eisend, 2006; Faison, 1961). Two-sided messages lead to a deeper message scrutiny and a more accurate focus on the message content than one-sided messages (Crowley \& Hoyer, 1994; Eisend, 2006; Faison, 1961; Kamins \& Assael, 1987). Hence, because of this deeper message scrutiny, we expect that the message content will be remembered more when the message is two-sided instead of one-sided.

\section{H.2 A two-sided anti sun tanning messages generates a higher message content recall than a one-sided anti sun tanning message.}

Under conditions of more elaborate message processing, individuals are more likely to engage in issue-relevant thinking by carefully evaluating the arguments in the message (Petty \& Cacioppo, 1984). Hence, when the message is two-sided instead of one-sided, more emphasis is put on the particular arguments in the message (Crowley \& Hoyer, 1994; Eisend, 2006). In other words, since a two-sided message's content is more thoroughly scrutinized than a onesided message's content, the relevance of the arguments used in the message is expected to be more influential in a two-sided message than in a one-sided message.

The present study investigates appearance versus health focused arguments about sun tanning. The relevance of arguments depends on the particular motive underlying the behavior or the issue at hand (Pham, 1998). Several studies have shown that sun tanning is primarily driven by aesthetical rather than health motivations (Heckman et al., 2009; Jones \& Leary, 1994; Turrisi et al., 1998). For instance, ample studies have found that individuals with a high appearance motivation are generally more prone to risky sun tanning behavior than individuals with a low appearance motivation (Shoveller et al., 2003; Leary et al., 1997; Heckman et al., 2009). Leary et al. (1997) found that high appearance motivated respondents most strongly endorsed the importance of having a tan, spent more time in the sun, were least 
likely to use sunscreen and were more likely to use tanning beds (Leary et al., 1997). It thus seems that individuals mainly suntan for appearance reasons.

If sun tanning is inherently driven by appearance motives, rather than health motives, appearance focused arguments will be more relevant, and thus more effective, than health focused arguments. More precisely, we expect a difference between one- and two-sided (processing depth): two-sided appearance focused messages against sun tanning will be more effective than their health focused counterparts. When a message is one-sided, however, the impact of argument focus (i.e., appearance versus health focus) is expected to be minor, as individuals focus less on the message content (i.e., the issue-relevance of the arguments), in a one-sided message than in a two-sided message. We therefore expect that:

H.3 When an anti sun tanning message is two-sided, an appearance focus leads to lower intentions to suntan than a health focus. This effect is expected to be less strong when an anti sun tanning message is one-sided.

\section{METHOD}

\section{Design and Stimuli}

A 2 × 2 between-subject factorial design manipulated message sidedness (one-sided versus two-sided) and argument focus (health versus appearance). Message sidedness was manipulated through different arguments: one argument against sun tanning versus the combination of one argument against and one argument in favor of sun tanning. The focus of the arguments was manipulated by the types of arguments chosen: "Sun tanning helps to maintain vitamin D levels, but causes skin cancer" (health focused argument) and "Sun tanning gives you an attractive tanned skin, but causes wrinkles" (appearance focused argument). Apart from the manipulations, the four ads were the same.

The stimuli were pretested among 60 respondents. The same measurement scales as in the main study were used (see hereafter). The results show that the respondents rated the twosided message $(M=6.49)$ as more two-sided than the one-sided message $(M=1.45)$ on a seven-point semantic differential scale $(t(77)=20.75, p<.001)$. The appearance focused message $(M=6.77)$ scored significantly higher on the seven-point health versus appearance scale than the appearance focused message $(M=1.50),(t(77)=29.99, p<.001)$.

\section{Participants and Procedures}

A total of 304 subjects participated in the study (69.5\% females, age range 18-65 years). The study deliberately targeted a wide age group, as sun tanning is not limited to a certain age group. The data were collected by means of an online questionnaire. The participants were randomly assigned to one of the four experimental conditions. The respondents received an email invitation to participate in the study, containing a link to the online questionnaire. The respondents were asked to forward the e-mail online to friends and acquaintances, hence the dispersion of the e-mail was based on the snowball method. Prior to exposure to one of the stimuli, respondents' appearance and health focus, attitude toward sun tanning and involvement with sun tanning were measured. Next, each respondent was individually exposed to one of the four stimuli. Subsequently, they completed the questionnaire, containing the manipulation check, followed by the dependent variables (i.e., attention to the message, recall of the message content, and behavioral intentions to suntan), age and gender. Finally, they were debriefed and thanked for their cooperation.

\section{Measures}


In line with previous studies (e.g., Eisend, 2006), message sidedness was measured by means of one single item on a seven-point semantic differential scale ("This message only gives arguments against sun tanning" vs. "This message gives arguments against sun tanning but also considers the arguments in favor of sun tanning"). Perceived appearance (versus health) focus of the message was measured by one single item on a seven-point semantic differential scale in analogy with previous studies (e.g., Jones \& Leary, 1994) (i.e., "This message emphasizes the effects of sun tanning on one's health" versus "This message emphasizes the effects of sun tanning for one's appearance"). Respondents' self-reported attention towards the message was assessed by five items on a seven-point Likert scale (Laczniak et al., 1989) (e.g., "I paid a lot of attention to the message.") $(\alpha=.88)$. Message content recall was measured by means of an open question in which the respondents were asked to list all content they remembered from the message, ranging from 1 (nothing) to 7 (all content). Prior attitude towards the issue was measured by means of a six-item seven point semantic differential scale (e.g., "I think sun tanning is negative versus positive") (Burgoon et al., 1997) $(\alpha=.94)$. Prior involvement with the issue was measured by four items on a seven point Likert scale by Beatty and Talpade (1994) (e.g., "I generally have a strong interest in sun tanning") ( $\alpha=.88)$. Respondents' health and appearance focus was measured by means of two separate subscales (Lawrence et al., 2006). The health focus subscale contained eight items on a seven-point Likert scale (e.g., "I am worried about health risks and take preventive action") $(\alpha=.79)$. The appearance focus subscale contained nine items on a seven-point Likert scale (e.g., "My looks are important to $m e$ ") $(\alpha=.87)$. Behavioral intentions to suntan were measured by means of three items on a seven-point semantic differential scale (Zhang \& Buda, 1999) (e.g., "How likely is the chance you will suntan?" ranging from 1 (very unlikely, not probable) to 7 (very likely, very probable) $(\alpha=.97)$.

\section{RESULTS}

\section{Manipulation Check}

A manipulation check shows that the appearance message $(M=4.70, S D=2.07)$ is considered more appearance (versus health) than the health message $(M=2.28, S D=1.59),(t(301)=$ $\left.11.48, \eta^{2}=.304, p<.001\right)$. Also, the two-sided message $(M=5.65, S D=1.54)$ was perceived as more two-sided than the one-sided message $(M=2.41, S D=1.56),\left(t(299)=18.15, \eta^{2}=\right.$ $.524, p<.001)$.

\section{Test of the Hypotheses}

Hypothesis $1 \mathrm{a}$ and $1 \mathrm{~b}$ were each tested by means of an independent samples t-test. The results show that a two-sided message $(M=4.55, S D=1.14)$ attracts more attention than a one-sided message $(M=3.85, S D=1.33),\left(t(298)=4.89, \eta^{2}=.074, p<.001\right)$, which confirms hypothesis 1. Additionally, we found that a two-sided message $(M=4.69, S D=1.51)$ generates a higher message content recall than a one-sided message $(M=3.83, S D=1.85),(t$ $\left.(302)=4.43, \eta^{2}=.061, p<.001\right)$, supporting hypothesis 2 .

Next, we tested in our sample the assumption that sun tanning is driven by appearance motivations rather than health motivations, by means of Pearson correlation analyses. A positive prior attitude toward sun tanning is positively correlated with individuals' appearance focus $(r(293)=.22, p<.001)$, while it is negatively correlated with a health focus $(r(295)=-$ $.22, p<.001)$. High involvement with sun tanning is also positively correlated with individuals' appearance focus $(r(293)=.25, p<.001)$, while it is uncorrelated with a health focus $(r(300)=.039, p=.507)$. Hence, sun tanning is generally more associated with appearance than health motivations. 
Hypothesis 3 was assessed through a one-way analysis of variance (ANOVA). The results show a significant interaction effect between message sidedness (one- versus two-sided) and argument focus (appearance versus health) on behavioral intentions to suntan $(F(1,290)=$ 4.266, $\eta^{2}=.015, p=.040$ ). Respondents indicate significantly lower behavioral intentions to suntan when they were given an appearance focused two-sided message $(M=2.88, S D=$ 2.11) than a health focused two-sided message $(M=3.71, S D=2.08), t(144)=2.39, \eta^{2}=$ $.038, p=.018)$, while for a one-sided message, no significant difference in behavioral intentions to suntan was found $\left(M_{\text {appearance }}=3.78, S D_{\text {appearance }}=1.99\right.$ versus $M_{\text {health }}=3.61$, $S D_{\text {health }}=2.12, t(143)=.522, \eta^{2}=.002, p=.603$ ) (see figure 1 ). Hypothesis 3 is supported.

\section{DISCUSSION, CONCLUSIONS AND FURTHER RESEARCH}

The present study investigated the effectiveness of health and appearance focus in one- and two-sided messages. The results show that two-sided messages attract more attention than one-sided messages. The content of two-sided messages is also better recalled than the content of one-sided messages. These results align with previous literature stating that twosided messages are generally processed in a more thorough manner than one-sided messages (Crowley \& Hoyer, 1994).

Furthermore, our results are in line with the previous studies that also used appearancefocused interventions next to the more conventional health focused messages. For instance, Jones and Leary (1994) found that a message emphasizing the negative consequences of UV exposure for future appearance was more effective in motivating intentions to engage in protective behaviors than was a message emphasizing the negative health consequences.

The added value of our study is the finding that argument relevance is especially important in two-sided rather than one-sided messages discouraging sun tanning. In a two-sided message, more emphasis is put on the arguments, because two-sided messages generally induce more thorough processing of the message content.

One of the implications of this study for health prevention practitioners is that two-sided messages should be implemented with caution: as the likelihood of more profound processing increases with two-sided messages, argument relevance becomes more important. Hence, when using two-sided message formats, practitioners should pay close attention to the relevance of the arguments they use for the issue at hand, even more so than when using onesided messages.

One limitation of this study is the omission of a control group. Further research could include a control group receiving a neutral message about sunbathing. Another suggestion would be to design a message in which both an appearance and a health focus are mixed. For instance, the arguments in favor of sun tanning might be appearance focused, whereas the arguments against sun tanning might be health focused. Additionally, besides health or appearance focused motivations, other motivations for sun tanning can be tested, such as the pleasure, feeling of joy, or warmth of sun tanning itself (i.e., consummatory motive) as opposed to sun tanning for a specific further goal (i.e., instrumental motive).

Further research could also investigate the effectiveness of promoting alternative behaviors, such as using self-tan creams or lotions. According to the theory of alternative behavior (Jaccard, 1981), attitude changes are optimal when alternatives are given for the discouraged behavior. In the present study, no alternative behaviors were mentioned in the messages.

Lastly, it would be interesting to assess age differences in further studies. Motivations for sun tanning as well as reactions to sun tanning prevention messages could vary in different age groups. 


\section{REFERENCES}

Ahn, H.Y., Wu, L., Kelly, S., \& Haley, E. (2011). A qualitative study of college student responses to conflicting messages in advertising: anti-binge drinking public service announcements versus wine promotion health messages, International Journal of Public Health, 56 (3), 271-279.

Arthey, S., \& Clarke, V.A. (1995). Suntanning and sun protection: a review of the psychological literature, Social Science \& Medicine, 40 (2), 265-274.

Beatty, S.E., \& Talpade, S. (1994). Adolescent influence in family decision making: a replication with extension, Journal of Consumer Research, 21, 332-341.

Belch, G.E. (1981). An examination of comparative and noncomparative television commercials: The effects of claim variation and repetition on cognitive response and message acceptance, Journal of Marketing Research, 18 (August), 333-349.

Burgoon, M., Cohen, M., Miller, M. D., \& Montgomery, C. L. (1978). An empirical test of a model of resistance to persuasion, Human Communication Research, 5, 27-39.

Crowley, A.E., \& Hoyer, W.D. (1994). An integrative framework for understanding two sided persuasion, Journal of Consumer Research, 20 (4), 561-574.

Devlin, E., Eadie, D., Stead, M., \& Evans, K. (2007). Comparative study of young people's response to anti-smoking messages, International Journal of Advertising, 26 (1), 99128.

Eisend, M. (2006). Two-sided advertising: a meta-analysis, International Journal of Research in Marketing, 23 (2), 187-198.

Eisend, M. (2007). Understanding, two-sided persuasion: an empirical assessment of theoretical approaches, Psychology \& Marketing, 24 (7), 615-640.

Faison, E.W.J. (1961). Symbology, Journal of Marketing, 25 (4), 119-119.

Ford, L.A., \& Smith, S.W. (1991). Memorability and persuasiveness of organ donation message strategies. American Behavioral Scientist, 34, 695-711.

Heckman, C.J., Wilson, D.B., \& Ingersoll, K.S. (2009). The influence of appearance, health, and future orientations on tanning behavior, American Journal of Health Behavior, 33 (3), 238-243.

Hoegh, H.J., Davis, B.D., \& Manthe, A.F. (1999). Sun avoidance practices among nonHispanic white Californians, Health Education \& Behavior, 26 (3), 360-368.

Hovland, C.I. (1954). Effects of the mass media of communication. In G. Lindzey (Ed.), Handbook of Social Psychology. Cambridge, MA: Addison-Wesley Publishing Company.

Jaccard, J. (1981). Attitudes and behavior: Implications of attitudes toward behavioral alternatives, Journal of Experimental Social Psychology, 17(3), 286-307.

Jones, J.L., \& Leary, M.R. (1994). Effects of appearance-based admonitions against sun exposure on tanning intentions in young adults, Health Psychology, 13 (1), 86-90.

Kamins, M.A., \& Assael, H. (1987). Two-sided versus one-sided appeals: a cognitive perspective on argumentation, source derogation, and the effect of disconfirming trial on belief change, Journal of Marketing Research, 24 (1), 29-39.

Laczniak, R.N., Muehling, D.D., \& Grossbart, S. (1989). Manipulating message involvement in advertising research, Journal of Advertising, 18 (2), 28-38.

Lawrence, J.W., Fauerbach, J.A., \& Thombs, B.D. (2006). A test of the moderating role of importance of appearance in the relationship between perceived scar severity and body-esteem among adult burn survivors, Body Image, 3 (2), 101-111.

Leary, M. R., Saltzman, J. L., \& Georgeson, J C. (1997). Appearance motivation, obsessive 
compulsive tendencies, and excessive suntanning in a community sample, Journal of Health Psychology, 2, 493-499

Ley, P., Witworth, M., Woodward, R., \& Yorke, R. (1977). The effect of sidedness and fear arousal on willingness to participate in a slimming scheme, Health Education Journal, 36, 67-69.

Pechmann, C. (1992). Predicting when two-sided ads will be more effective than one-sided ads: the role of correlational and correspondent inferences, Journal of Marketing Research, 29 (4), 441-453.

Petty, R.E., \& Cacioppo, J.T. (1984). The effects of involvement on responses to argument quantity and quality: Central and peripheral routes to persuasion, Journal of Personality and Social Psychology, 46 (1), 69-81.

Pham, M. T. (1998). Representativeness, relevance, and the use of feelings in decision making, Journal of Consumer Research, 25 (2), 144-159.

Shoveller, J.A., Lovato, C.Y., Young, R.A., Moffat, B. (2003). Exploring the development of sun-tanning behavior: a grounded theory study of adolescents' decision-making experiences with becoming a sun tanner, International Journal of Behavioral Medicine, 10 (4), 299-314.

Turrisi, R., Hillhouse, J., \& Gebert, C. (1998). Examination of cognitive variables relevant to sunbathing, Journal of Behavioral Medicine, 21 (3), 299-313.

Zhang Y., \& Buda, R. (1999). Moderating effects of need for cognition on responses to positively versus negatively framed advertising messages, Journal of Advertising, 28, $1-15$. 
behavioral intentions to suntan

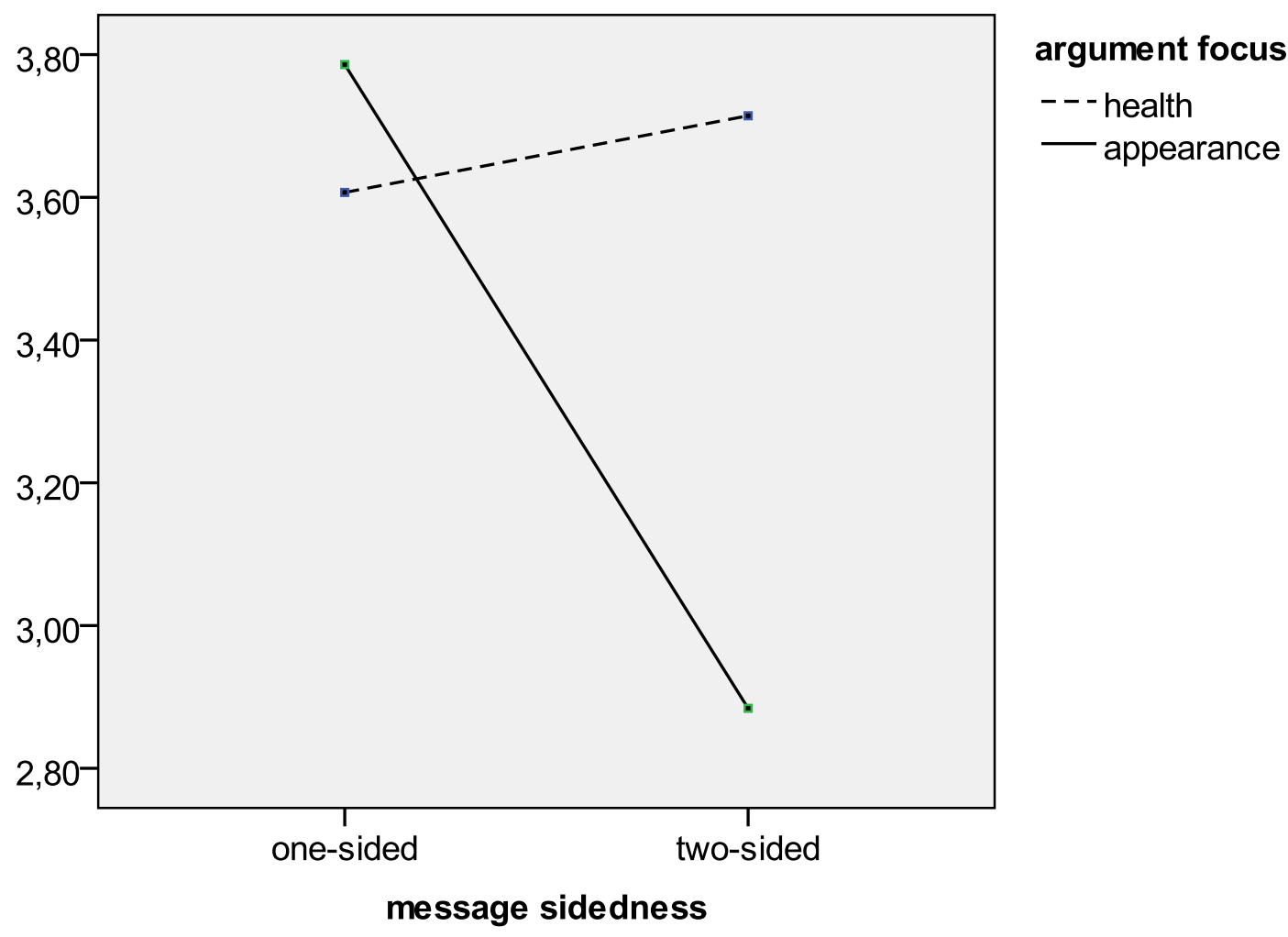

Figure 1: The interaction effect between message sidedness and argument focus on behavioral intentions to suntan. 